\title{
Pratiques
}

Linguistique, littérature, didactique

141-142 | 2009

La synonymie

\section{La synonymie - « identité de sens » n'est pas un mythe}

Georges Kleiber

\section{(2) OpenEdition}

1 Journals

Édition électronique

URL : https://journals.openedition.org/pratiques/1262

DOI : 10.4000/pratiques.1262

ISSN : 2425-2042

Éditeur

Centre de recherche sur les médiations (CREM)

Édition imprimée

Date de publication : 15 juin 2009

Pagination : 9-25

Référence électronique

Georges Kleiber, "La synonymie - « identité de sens » n'est pas un mythe », Pratiques [En ligne],

141-142 | 2009, mis en ligne le 19 juin 2014, consulté le 10 mars 2023. URL : http://

journals.openedition.org/pratiques/1262 ; DOI : https://doi.org/10.4000/pratiques.1262

Tous droits réservés 


\section{La synonymie-« identité de sens » n'est pas un mythe}

\section{Georges Kleiber}

Université de Strasbourg \& EA 1339 LILPA-Scolia

Much research remains to be done in the field of synonymy (Cruse, $2000: 161$ )

\section{Introduction : le paradoxe de la synonymie}

La synonymie est vraiment une relation sémantique paradoxale. C'est la relation la plus facile à aborder et la plus facile à définir, mais aussi la plus difficile à maintenir. Quasiment tout le monde est d'accord pour la définir comme une identité ou équivalence de sens entre deux unités lexicales différentes, mais quasiment tout le monde aussi s'accorde pour dire qu'une telle identité ou équivalence sémantique ne se trouve pas réellement et donc que la vraie synonymie au fond n'existe pas ou - pour les plus prudents - « paraît, pour le moins, extrêmement rare » (Nyckees, $1998: 181)$. Il est ainsi piquant de noter que le but principal des dictionnaires de synonymes n'est pas tellement de montrer l'identité sémantique des lexèmes retenus comme synonymes, mais consiste précisément à mettre au jour leurs différences, si minimes soient-elles. Et de nombreux articles de linguistique, même et surtout dans les recueils consacrés à la synonymie, suivent ce mouvement : 1'auteur prend deux ou trois expressions considérées habituellement comme synonymes et déploie ensuite tout son talent de fin sémanticien pour prouver qu'il n'en est finalement rien, que chaque expression a son sens et son fonctionnement propres, qui conduisent à rejeter in fine l'assimilation sémantique " grossière » qui a servi de point de départ.

Bien sûr qu'on ne jette pas les bébés synonymes avec l'eau du bain dissimilateur ! Si l'on renonce à l'existence d'expressions différentes qui sont totalement identiques du point de vue de leur sens, on ne reverse pas pour autant les candidats synonymes recalés dans le lot commun des vocables de sens différent. On tient malgré tout à leur conserver un statut sémantique spécial qui rend compte de l'intuitive proximité sémantique qui est à l'origine de leur candidature à la synonymie. Et l'on parle alors à leur propos - les termes varient dans la littérature - de pa- 
rasynonymes, de synonymie large, relative, partielle, incomplète, approximative ou encore de quasi-synonymie, ou même, en recourant à l'oxymore, de synonymie distinctive $^{(1)}$, c'est-à-dire d'unités lexicales dont le sens est presque identique ou dont les différences sémantiques n'arrivent pas à supplanter ce qu'ils ont de sémantiquement identique.

Ce statut spécial de quasi-synonyme, qui peut donner lui-même lieu ou non à une sous-classification qualitative ${ }^{(2)}$, conduit à requalifier la relation d'identité de sens, à la base de la notion de synonymie, comme étant une relation de synonymie absolue, totale, parfaite, complète ${ }^{(3)}$ étroite, stricte, exacte ou encore de vraie synonymie et offre une porte de sortie au paradoxe initial d'une synonymie qui existe et n'existe pas. C'est la synonyme absolue qui n'existe pas ou qu'exceptionnellement. Il ne s'agit que d'un mythe ou d'un leurre et, en sémantique lexicale, affirmer que la vraie synonymie ou synonymie absolue, complète ou totale ne se rencontre pas est devenu une vulgate répétée, depuis 1'abbé Girard et ses Synonymes François ${ }^{(4)}$, par la majorité des commentateurs :

«Je ne crois pas qu'il y ait de mots synonymes dans aucune Langue... je suis très persuadé et j'ose dire assuré qu'il n'y en a point dans la nôtre » (Abbé Gabriel Girard, 1718, Préface $1^{\text {re }}$ édition, xxx, cité par Martin, 1976 : 115).

«Pour acquérir la justesse, il faut se rendre un peu difficile sur les mots : ne point s'imaginer que ceux qu'on nomme synonymes le soient dans toute la rigueur d'une ressemblance parfaite, en sorte que le sens soit aussi uniforme entre eux que l'est la saveur entre les gouttes d'eau d'une même source. Car en les considérant de près, on verra que cette ressemblance n'embrasse pas toute l'étendue et la force de la signification. [...] La ressemblance que produit l'idée générale fait donc les mots synonymes; et la différence qui vient de l'idée particulière qui accompagne la générale, fait qu'ils ne le sont pas parfaitement et qu'on les distingue comme les diverses nuances d'une même couleur » (Abbé Gabriel Girard, $1769^{(5)}$, Préface, vii, cité par Batteux, $2000: 13$ )

« C'est presque un truisme de dire que la synonymie totale est un phénomène extrêmement rare, un luxe que la langue ne peut se permettre (Ullmann, 1952 : 38)

«On peut cependant affirmer qu'il n'existe pas de vrais synonymes, qu'il n'existe pas deux mots ayant exactement le même sens » (Baylon et Fabre, 1978 : 168)

«L'identité de sens (ou synonymie absolue) est un leurre » (Lehmann et MartinBerthet, $1998: 54)$

«Il ne semble donc pas exister véritablement de synonymes absolus » (Nyckees, $1998: 181)$

(1) Voir, par exemple, le titre de l'article récent de Berlan (2007): «Peut-on concilier la notion de valeur et le recours à l'étymologie en synonymie distinctive?».

(2) La plupart des manuels de lexicologie (Picoche, 1993, Mortureux, 1997, Lehmann et Martin-Berthet, 1998; Gaudin et Guespin, 2000 et Polguère, 2008) ou de sémantique (Baylon et Fabre, 1978, Brekle, 1974, Tamba, 1988, Nyckees, 1998 et Touratier, 2000) se contentent d'une division binaire en synonymie absolue / parasynonymie. On trouvera chez Lyons (1970), Martin (1976), Mel'Cuk, Clas et Polguère (1995) et Cruse (2000) des subdivisions qualitatives du côté des parasynonymes.

(3) Martin (1976) utilise les épithètes de totale et de complète de deux manières différentes.

(4) Il s'agit du titre de la $2^{\mathrm{e}}$ édition (1736). Le titre de la première édition (1718), révélateur, est La Justesse de la langue françoise ou les différentes significations des mots qui passent pour synonymes.

(5) Il s'agit de la préface de la $3^{\mathrm{e}}$ édition. 
« Il semble qu'il n'y ait jamais de synonymie parfaite » (Siouffi et Van Raemdonck, 1999 : 109)

«Il est essentiel de noter que la synonymie lexicale exacte est extrêmement rarissime [...] La synonymie lexicale est avant tout une synonymie approximative. » (Polguère, 2008: 150)

Cette solution d'une synonymie qui n'exige plus une identité sémantique complète n'a plus rien de contradictoire, mais elle reste néanmoins paradoxale, puisqu'on n'abandonne pas pour autant le cas de la synonymie absolue où les items synonymes auraient exactement le même sens. Pourquoi conserver, comme le font quasiment toutes les présentations, cette définition en termes d'identité de sens, si c'est pour dire qu'elle n'a aucune réalité langagière et que seuls co-existent des termes en synonymie relative ${ }^{(6)}$ ? N'y aurait-il pas là anguille sous roche ? Autrement dit, ne serait-ce pas parce qu'elle possède malgré tout une certaine pertinence linguistique qu'elle continue de s'imposer dans le champ des relations de sens lexicales?

Notre contribution se limitera à examiner les différentes facettes de ce problème. Nous essaierons de montrer contre vents et marées non synonymiques que l'identité de sens, c'est-à-dire la synonymie tout court, existe réellement. Si la remettre en cause est devenu un truisme, c'est tout simplement parce que l'argumentation employée pour la nier présente des côtés fallacieux, repris quasiment par tous nos devanciers, qui conduisent à ce résultat.

Nous présenterons donc successivement les trois principaux arguments avancés pour rejeter l'existence de lexèmes «complètement» synonymes et essaierons à chaque fois de mettre au jour ce qui en mine la pertinence. Le premier argument, qui sera examiné dans la première partie, se situe au niveau général de l'économie linguistique. Il revient à nier que le système sémiotique d'une langue puisse tolérer de véritables synonymies. Dans notre deuxième partie, nous aborderons le deuxième, qui se place au niveau de la nature du sens même en distinguant deux composantes sémantiques dont l'une serait spécifique à chaque unité lexicale. Enfin, dans notre troisième et dernière partie, sera traité l'argument qui touche plus spécialement la manifestation ou reconnaissance de la synonymie absolue, celui de l'interchangeabilité totale.

Qu'on ne se méprenne point sur notre entreprise, il ne s'agit pas pour nous de rejeter l'existence de quasi-synonymes - c'est un autre problème que nous ne traiterons pas ici, mais qui mérite à coup sûr, comme le souligne Cruse (2000:161), que l'on s'y arrête plus longuement qu'on ne l'a fait jusqu'ici. La quasi-synonymie existe, bien entendu, et sans doute plus abondamment que la synonymie « absolue ». Notre objectif est d'aller en quelque sorte à contre-courant des opinions sémantiquement correctes en matière de synonymie en montrant que l'idée d'une synonymie, conforme à sa définition initiale, c'est-à-dire l' «identité de sens », ne se trouve finalement pas mise à mal par les assauts-critiques qu' on ne cesse de mener contre elle et qu'il ne s'agit donc pas seulement d'un mythe ou d'un leurre.

(6) Cf. la suggestion de Neveu $(2004: 281):$ «[... ne vaut-il pas mieux de s'en tenir à la définition large de la synonymie, ne serait-ce que pour s'accorder avec l'usage courant et éviter ainsi discussions ou malentendus inutiles?» 


\section{Arguments en relation avec la nature des langues}

\subsection{Tout signe est oppositif}

Si l'on accepte, dans une optique saussurienne, qu'une langue est un code où chaque signe a sa valeur définie par les relations différentielles qu'il entretient avec les autres signes, l'on est conduit tout logiquement à refuser l'idée de synonymie absolue, puisque des unités totalement synonymes n'entretiennent par définition nul rapport oppositif ou contrastif : «Si l'on suit fidèlement la leçon de Saussure, tout signe possédant une valeur négative, il n'existe pas de synonyme vrai, puisque toutes les unités s'opposent » (Gaudin et Guespin, 2000 : 177). C'est encore dire, d'une autre manière, avec Dumarsais (1730:236), cité par Tamba (1988: 83) que « s'il y avait des synonymes parfaits, il y aurait deux langues dans une même langue ».

L'argument ne tient véritablement que si tout signe est véritablement oppositif. Mais ceci demande à être prouvé, les synonymes représentant précisément un contre-argument à cette thèse. Et, surtout, rien n'interdit a priori de penser qu'une langue puisse posséder deux signes de même sens, c'est-à-dire deux signes reliés par une relation d'identité de signifié qui entrent tous deux dans le réseau oppositionnel à d'autres signes. Une course peut avoir deux vainqueurs dont la relation structurale «d'identité » est alors, bien évidemment, pertinente, comme l'est également leur relation oppositive aux deuxième, troisième, quatrième, etc., de la course.

\subsection{Toute différence de forme $=$ une différence de sens}

Un deuxième argument, qui prolonge le premier en lui apportant le fondement qui lui manque, consiste à postuler, sous une bannière aux couleurs guillaumiennes précognitivistes, que toute différence de forme se traduit par une différence de sens : «...each linguistic form has a constant and specific meaning. If the forms are phonemically different, we suppose that their meanings also are different " (Bloomfield, cité par Ullmann, 1962 : 82). La synonymie-« identité de sens »se voit évidemment par là-même placée hors jeu par avance. Cet argument est relié le plus souvent au sentiment des locuteurs qui, « lorsque la forme est différente [...] s'attendent comme le soulignent Lehmann et Martin-Berthet. (1998: 54), à une différence de sens ». Opinion que l'on retrouve chez Tamba (1988:83) qui note aussi que «l'idée d'une synonymie parfaite heurte le sens commun », parce que celui-ci « tend à apparier une différence de forme à une différence de sens ».

Mais là encore cette thèse qui veut qu'une différence de forme entraîne toujours une différence de sens demande à être démontrée et la synonymie peut à nouveau servir de contre-argument. Elle semble en tout cas excessive pour traiter des cas de simple variation formelle du type pharamineux / faramineux et ne semble pas appropriée pour rendre compte des variantes morphologiques du type je / me / moi, même s'il s'est trouvé des auteurs pour défendre l'idée que me est finalement un autre, c'est-à-dire nije ni moi (Leeman, 2002). Et si l'on se tourne du côté psychologique, il nous semble que, face à des synonymes classiques comme chaussure et soulier ou voiture et auto, les usagers ne sont pas choqués outre mesure par leur synonymie, mais acceptent comme allant de soi qu'ils signifient la même chose. En tout cas, à la différence du sémanticien, ils ne sont nullement poussés à chercher à tout prix une différence sémantique entre les deux. 


\section{3. «Inutilité ", « luxe ", « embarras » sémiotiques}

Une autre manière d'expliquer l'absence de véritables synonymes dans une langue est de mettre en avant leur inutilité sémiotique. C'est la seconde raison qu'invoque Dumarsais (1730:236, toujours cité par Tamba, 1988:83) pour expulser les synonymes " parfaits » : "Il est fort inutile d'avoir plusieurs mots pour une même idée », car c'est - l'expression est d'Ullmann (cité par Lyons, 1970 : 342) « un luxe que la langue ne peut se permettre ». C'est donc un principe même d'économie linguistique, que Neveu qualifie de «salutaire» (2004:281), qui serait à l'origine de la répugnance des langues à posséder de "vrais " synonymes. A quoi bon avoir des synonymes puisqu'il ne s'agit que de « doublons »? Et la richesse synonymique associée à certaines langues, s'il s'agit réellement de synonymie absolue, ne serait au fond qu'embarras de richesse comme on a pu le dire, par exemple, à propos de la (sur)abondance de synonymes qui caractérise l'ancien français (von Wartburg, $1969: 281$ ).

La preuve que les langues répugnent à l'inutilité ou au luxe « sémiotique » que représentent les Doppelgänger lexicaux, se trouve, comme le soulignent la plupart des commentateurs, dans la réaction d'élimination ou de spécification qu'elles manifestent devant l'existence de doublets acquis soit par emprunt, soit amenés par l'évolution en deux mots différents d'un même étymon : soit un des termes se trouve éliminé, soit il y a différenciation sémantique entre les deux (hôtel et hôpital,frêle et fragile). «De fait, écrivent Baylon et Fabre, 1978 : 168), il paraîtrait invraisemblable que deux mots ayant exactement le même sens survivent tous deux dans la langue ». Ce refus d'un maintien d'un " même " sémantique pour deux termes différents correspond chez Frei (1929) au besoin de différenciation et chez Bréal (1897) à la loi de répartition, que rappelle en note Tamba (1988: 84) : «Nous appelons répartition l'ordre intentionnel par suite duquel des mots qui devraient être synonymes et qui l'étaient en effet ne peuvent plus s'employer l'un pour l'autre». Par exemple, " entre l'estime, le respect, la vénération, on n'aperçoit nulle gradation imposée par l'étymologie. Il a fallu des esprits exacts et précis, une société ordonnée et soucieuse des rangs, pour établir certaines distinctions » (Bréal, 1897 : 26-38).

Il est évidemment impossible de nier que la synonymie absolue implique du « superflu » sémantique, mais cela ne signifie pas, ipso facto, qu'il s'agit d'une inutilité sémiotique, c'est-à-dire d'une inutilité au niveau des signes même et non pas seulement au niveau de leur sens. Lorsqu'on emploie un mot, ce n'est, bien entendu, pas seulement son sens qui se trouve utilisé, mais c'est bien le mot luimême, en entier, c'est-à-dire avec ses particularités formelles et sémantiques, qui se trouve placé dans la chaîne discursive. Du coup, même si au niveau du seul sens, l'existence de deux synonymes stricts peut apparaître comme étant un luxe sémémique, dès qu'on les envisage dans leur globalité et donc dans leur différentialité sémiotique - ce sont des mots différents - il apparaît clairement que, parce qu'ils peuvent avoir une fonctionnalité discursive différente du fait précisément de leur différence de «forme », cette co-existence synonymique, loin d'être un fardeau, s'avère être des plus précieuses pour leur insertion syntagmatique. Sur un saxophone aussi, il existe des clés-« synonymes », c'est-à-dire des clés qui donnent le même son. Leur utilité fonctionnelle devient pourtant claire pour peu que l'on se mette à jouer de l'instrument.

Il en va de même selon nous des synonymes « absolus ». On pourrait d'abord mentionner ici la dimension mémorielle et avancer l'argument que la possession de dou- 
blons sémantiques permet de pallier à d'éventuelles pannes de performances dénominatives neuronales, mais cette dimension, que l'on aurait tort de sous-estimer, est encore trop peu connue pour que l'on se risque à émettre des hypothèses qui soient un tant soit peu sérieuses. Ce qui est plus sûr, par contre, c'est que les propriétés «formelles »-par définition différentes - de deux synonymes stricts se révèlent être des éléments fort utiles au moment de l'insertion syntagmatique : les facteurs prosodiques ${ }^{(7)}$ et morphologiques ${ }^{(8)}$ jouent à l'évidence un rôle qui, sans toujours être prépondérant - il peut se cantonner au niveau stylistique - n'en est pas moins précieux. Sans compter d'autres facteurs d'usage, qualifiés de « connotatifs» qui s'attachent aux mots et que nous laissons pour le moment de côté, afin de ne pas brouiller notre raisonnement, mais sur lesquels nous reviendrons ci-dessous. Signalons simplement encore que, sur le plan textuel, la synonymie absolue constitue le moyen idéal d'échapper à la répétition lexicale, si l'on considère qu'une reprise lexicale fidèle représente ${ }^{(9)}$ un «moins » pour la réussite discursive. Un des deux résultats consignés par Lehmann et Martin-Berthet (1998 : 58) dans leur conclusion à la section consacrée à la synonymie porte précisément sur ce point : «La synonymie se distingue des autres relations sémantiques par deux points : la forte dépendance par rapport au contexte et ses liens avec les contraintes d'ordre stylistique (l'utilisation des synonymes est recommandée pour éviter l'abus de répétitions) ».

Qu'en est-il alors de l'argument diachronique qui veut la « mort» ou le changement pour l'un des deux (ou les deux) synonymes absolus ? On apportera une réponse en deux parties. Tout d'abord, nous ferons valoir que des exemples tels que chaussure / soulier, voiture / auto, vélo / bicyclette, etc., sont des contre-exemples marquants à une telle évolution. On nous objectera, sans doute, qu'ils ne sont pas totalement synonymes. Acceptons-le provisoirement. Mais, et c'est la seconde partie de notre réponse, quelle que soit cette différenciation, elle n'est pas du type de celle que l'on avance habituellement pour illustrer la spécialisation sémantique que subit l'un des doublets. Les exemples d'évolution anti-synonymique avancés sont tous du type

(7) Voir par exemple, Lyons (1970:344) : "Dans la composition des vers, les restrictions en matière de phonologie qu'imposent le rythme et l'agencement des rimes constituent une autre influence non sémantique sur la sélection des mots ».

(8) Pour ce qui est de la syntaxe, c'est un autre problème, puisque la synonymie est généralement considérée comme ne s'établissant qu'entre deux mots de catégorie grammaticale identique. Cette condition reste généralement implicite. Elle se trouve explicitée chez Tamba (1988: 81) qui rappelle que « dans l'usage courant, on appelle synonymes des mots différents de même catégorie grammaticale et de sens à peu près équivalent » et chez Sanchez et Cadiot (2007: 138) qui soulignent aussi que « la condition préalable à tout rapprochement synonymique » est « 1 'appartenance des lexèmes à une même classe grammaticale ». On comprend la raison de cette exigence : la différence de catégorie grammaticale impliquant normalement une différence de sens, l'identité de sens totale n'est d'avance plus acquise. Si l'on en fait abstraction, l'on peut obtenir, dans une perspective onomasiologique, des synonymes de catégorie grammaticale différente. «Il reste, observe Mitterand (1963: 77), qu'un signifié unique peut se trouver exprimé par une quantité variable de mots différents compte tenu des servitudes morphologiques, syntagmatiques et stylistiques qui pèsent sur l'emploi des mots ». Et il ajoute qu'il « semble donc possible de dessiner la table synonymique de l'emploi de ces mots ». La dimension fonctionnelle de la synonymie apparaît alors encore beaucoup plus fortement, puisque la différence de catégorie grammaticale des synonymes présente des alternatives syntaxiques là où l'identité de catégorie grammaticale synonymique n'offre qu'un seul choix de construction syntaxique possible.

(9) Ce facteur, c'est-à-dire le désir d'éviter la répétition, est un argument commode, bien souvent avancé trop facilement, à nos yeux, dans les argumentations linguistiques. En matière de synonymie étroite comme large, il s'impose beaucoup plus naturellement. 
hôpital / hôtel, à savoir qu'ils aboutissent à deux mots qui sortent de la problématique synonymique en ce qu'ils ne peuvent même plus être considérés comme des parasynonymes ou quasi-synonymes. Or, les couples que nous avons cités restent, pour tous les commentateurs, dans l'aire de la synonymie, large, certes, mais de la synonymie quand même. L'argument diachronique n'est donc pas plus décisif que celui de l'inutilité sémiotique qu'il est censé confirmer. Des synonymes absolus ne sont pas forcément des tares pour le système linguistique : ils ont une utilité discursive certaine, à partir du moment où l'on considère que ce ne sont pas seulement des sens qui sont intégrés dans le discours, mais bien des mots dont les spécificités font qu'ils ont aussi leur... mot à dire dans cette intégration.

\section{Deux types de sens}

\subsection{Sens dénotatif et sens connotatif}

Beaucoup plus sérieux pour condamner la synonymie absolue est le recours au type de sens. Il est devenu courant de refuser la synonymie absolue au nom de la différence de sens « connotatif» ou encore de « sens affectif» ${ }^{(10)}$. Cette condamnation repose sur la division préalable du sens lexical en deux composantes : une composante appelée cognitive ou dénotative, qui correspond en gros à la structure sémique de la sémantique structurale ou au sens vériconditionnel ${ }^{(11)}$ (ou encore référence « virtuelle » chez Milner ou sens « catégoriel » chez Tamba, 1988) de la sémantique logique et une composante nommée connotative ou affective, assez hétéroclite ${ }^{(12)}$, qui rassemble les différents traits subjectifs, expressifs, linguistiques, tous jugés non essentiels ou non distinctifs, mais qui se trouvent néanmoins associés à une unité lexicale. Il suffit alors de postuler, comme le fait par exemple Rey-Debove (1997), que tout mot possède un sens connotatif qui est différent de celui des autres unités lexicales pour que la synonymie absolue soit exclue par avance. Dans une telle théorie de la signification, il ne peut plus y avoir de synonymes absolus dans la mesure où tout mot possède " une partie désignative qu'il peut avoir en commun avec d'autres mots et une partie connotative propre qui ne se retrouve dans aucun autre mot» (Rey-Debove, 1997 : 98). C'est dire avec Lehmann et Martin-Berthet (1998: 55) que « lorsque les mots synonymes ont le même sens dénotatif, ils diffèrent par leurs composantes pragmatiques ou signifié connotatif ».

Les composantes de ce signifié connotatif spécifique à chaque unité lexicale sont bien connues des spécialistes et des usagers des dictionnaires. La terminologie et leur nombre peut varier selon les présentations. Baldinger (1984:187) n’en distingue pas moins de 23 ! Nous citerons ici pour illustration la présentation de Lehmann et Martin-Berthet (1998: 57) :

«Les différences pragmatiques des synonymes renvoient à différents aspects de la variation lexicale, traitée dans le dictionnaire sous la forme des marques d'usage (Vx, Fam., Litt., etc.) :

- variations diachroniques : bru / belle-fille, épatant / super ;

- variations géographiques (dites aussi diatopiques) : wassingue (nord de la

(10) Les deux termes ne sont pas tout à fait équivalents; le premier est beaucoup plus large que le second.

(11) Le péché, impardonnable !, consisterait à parler de sens « référentiel».

(12) Lyons la qualifie de «fourre-tout» (1970:344). 
France) ou panosse (dans le Midi) / serpillière ;

- variations liées aux registres de langue (dites diastratiques) : familier ou populaire / standard (futal / pantalon, tronche / tête) littéraire / standard (croisée / fenêtre), argot / langue commune (meuf / femme, keuf / flic) ;

- variations liées à l'opposition langues de spécialité / langue commune :rhinite/ rhume, préposé / facteur ;

- connotations, qu'il s'agisse de péjoration (nègre par rapport à noir ou black) ou au contraire d'euphémismes : longue maladie / cancer, demandeur d'emploi pour chômeur, technicien de surface pour balayeur, SDF pour sans-abri ».

Appliquée au triplet vélo, bicyclette, bécane, cette approche conclura à leur sens dénotatif identique, mais leur reconnaîtra des sens connotatifs différents : bécane sera marqué familier et vélo, à la différence de bicyclette, apparaît associé au cyclisme, ainsi que le rapportent Gaudin et Guespin (2000 : 178) : « dans le domaine sportif, on utilise vélo: on parlera de vélo de course et non de ? bicyclette de course. On qualifiera Miguel Indurain de roi du vélo; roi de la bicyclette ferait figure de plaisanterie $»$.

Le tour est ainsi joué : le recours au salvateur signifié connotatif permet de se sortir du paradoxe initial, pour une bonne partie du moins des cas de synonymie ${ }^{(13)}$, puisqu'il établit à la fois l'identité, au niveau du sens dénotatif, et la distinctivité, au niveau du sens connotatif. Pour Tamba (1988 : 85), la synonymie ne se situe donc pas sur le plan du sens dénotatif, mais sur celui du sens connotatif en ce qu'elle « ne peut que surajouter un trait connotatif à une dénotation préétablie ». Le sort de la synonymie absolue se trouve par là-même également scellé : le signifié connotatif la fait définitivement disparaître. Il n'y a plus de synonymes véritablement absolus, c'est-à-dire sémantiquement totalement identiques.

\subsection{Il ne s'agit pas de... sens}

Une telle issue peut, nous semble-t-il, être évitée, parce que l'argument du sens connotatif propre à chaque unité « synonymique » n'a pas la pertinence qu'on lui reconnaît et qui fait qu'il soit repris dans quasiment toutes les réflexions sur la synonymie. Il ne s'agit pas de remettre en cause l'existence de ce méli-mélo de traits ni d'essayer de trouver dans les recoins du vocabulaire des synonymes qui seraient des contre-exemples en ce qu'ils présenteraient des traits connotatifs totalement identiques. Ce n'est pas à ce niveau que le bât blesse. Là où il pèche, c'est au niveau de la caractérisation de ces traits : ces marques connotatives diachroniques, géographiques, diastratiques, expressives, etc., ne sont pas l'apanage du sens du mot synonymique, mais concernent le mot dans sa globalité et donc ne touchent nullement à l'identité sémantique qu'il peut y avoir ou non entre deux synonymes. Il n'y a donc pas lieu de parler à leur propos de signifié qu'il soit connotatif ou non. Ces marques indiquent simplement que les mots synonymes sont des mots qui se distinguent, non par leur sens ${ }^{(14)}$, mais par des caractéristiques qui sont attachées à leur statut de mot.

Comment le prouver? On commencera par un détour, en passant par la synonymie " interlinguistique» : arbre et Baum ont grosso modo le même sens, mais diffèrent quant à la langue. On ne dira pas pour autant que leur signifié ou sens conno-

(13) Il reste en effet les cas de synonymie à sens dénotatif différent.

(14) Ce qui sera le cas des synonymes à sens dénotatif différent (cas prévu dans les typologies de Lyons et de Martin). 
tatif est différent, qu'arbre a pour sens connotatif « français » et Baum « allemand », mais on notera qu' arbre est un mot français et Baum un mot allemand. Il n'y aurait aucun sens à affirmer que le sens (de quelque type qu'il soit) d'arbre est français, alors que celui de Baum est allemand. Il en va de même avec les différentes composantes du prétendu signifié connotatif des synonymes intralinguistiques. Les indications de type vieilli, familier, populaire, spécialisé, savant, littéraire, poétique, standard, argotique, vulgaire, langue de spécialité, langue commune, expressif, etc., qualifient le mot et non le sens du mot, parce que ce n'est pas le sens qui est vieilli, familier, populaire, etc., mais bien le mot ! Reprenons le synonyme bécane auquel on a assigné ci-dessus le sens connotatif « familier ». Ce n'est bien évidemment pas le sens qui est familier, celui de bicyclette et de vélo le sont tout autant, mais c'est le mot (tout entier) qui relève d'un emploi familier. On notera à cet égard que les attributs connotatifs se combinent mieux, en énoncé épilinguistique, à mot comme sujet qu'à sens :

Le mot $X$ est familier, savant, populaire, poétique, expressif, etc.

? Le sens de X est familier, savant, populaire, poétique, expressif, etc.

Il faut donc admettre que bon nombre de traits dont on a chargé la mule sémantique appartiennent en fait au mot lui-même. Ceci suppose évidemment que le mot puisse avoir des qualités propres, qui résultent certes de sa forme et de son sens et de la relation entre les deux ${ }^{(15)}$, mais qui s'attachent à l'unité conventionnelle (codée) formelle et sémantique qu'il constitue. Si on reprend sur cette base, la question des synonymes différant uniquement par des traits connotatifs, alors la synonymie absolue ou synonymie tout court, c'est-à-dire la synonymie-« identité de sens ", retrouve pleinement droit de cité, puisque les synonymes en question ne diffèrent plus par leur sens, mais par les caractères attribués aux mots différents qu'ils constituent. Il ne s'agit pas simplement d'une variation de signifiant, mais bien d'une variation lexicale, c'est-à-dire de mots différents. Ainsi la variation orthographique faramineux / pharamineux, déjà mentionnée ci-dessus, ne donne pas lieu à deux mots différents, mais à deux formes différentes d'un même mot; elle ne pourra donc prétendre au label synonymique.

On comprend par là-même pourquoi les analyses antérieures ont quasiment toutes été conduites à sacrifier la synonymie absolue qu'ils avaient posée comme point de départ : elles sont parties toutes de l'idée qu'une synonymie totale ne devait correspondre qu'à une simple variante de signifiant, c'est-à-dire à une simple variante de forme, puisque le sens était identique. Or, une telle position méconnaît les qualités propres à l'unité lexicale : si la forme d'un mot a ses spécificités et si le sens de ce mot a les siennes, le mot qui stabilise leur réunion a aussi les siennes qui ne se résument point en la seule addition des deux, dans la mesure où, point que nous avons déjà souligné et sur lequel nous reviendrons, c'est bien un mot qui se trouve employé dans le discours et non seulement un sens ou une forme.

Il n'y a donc plus rien de paradoxal en matière de synonymie si l'on se cantonne aux candidats à la synonymie absolue ${ }^{(16)}$ recalés pour différence de connotations : ces différences proviennent tout logiquement du fait qu'il ne s'agit pas de simple variantes formelles d'un même mot, mais aussi de mots différents, c'est-à-dire

(15) C'est ici qu'il conviendrait de développer deux problématiques différentes, celle de la dénomination et celle du symbolisme, de l'iconicité et de l'indexicalité sémiotiques.

(16) Rappelons une nouvelle fois encore qu'il en va évidemment différemment avec les synonymes à sens dénotatif différent. 
d'unités lexicales différentes. Il n'y a donc pas lieu de parler à leur propos de parasynonymes ou de quasi-synonymie ou encore de synonymes approximatifs. La meilleure preuve en est encore le test épilinguistique de avoir le même sens que / avoir presque le même sens que. On observe que l'énoncé :

"vélo » a le même sens que "bicyclette »

paraît indiscutablement mieux formé que l'énoncé :

? "vélo » a presque le même sens que "bicyclette "

le second suscitant une réaction du type mais ils ont le même sens !

Et si l'on osait (encore !) manipuler le critère référentiel on opposerait de la même manière les énoncés :

Un vélo, c'est la même chose qu'une bicyclette

? Un vélo, c'est presque la même chose qu'une bicyclette

\section{Interchangeabilité}

\subsection{Synonymie absolue $=$ substituabilité totale}

Nous n'en avons cependant pas encore fini avec la défense de la synonymie« identité de sens ». Il y a un troisième argument - de taille - qui, bien souvent, est avancé pour conclure à son inexistence, c'est celui de l'interchangeabilité totale. L'idée sous-jacente est que si deux mots différents sont sémantiquement totalement identiques, cette identité de sens doit aussi se manifester par une commutation totale dans leurs emplois. Si des mots sont des synonymes absolus, ils ne varient que par leur forme et donc doivent fonctionner à la manière des allomorphes en variation libre ${ }^{(17)}$, c'est-à-dire que là où se trouve l'un doit pouvoir se trouver 1'autre. Une telle relation entre sens et distribution repose sur l'idée admise quasiment par tous les sémanticiens que le sens d'une unité conditionne ses emplois, c'est-à-dire sa distribution, ses combinaisons syntagmatiques. Il s'ensuit (presque) tout logiquement que des synonymes absolus ou mots ayant le même sens doivent connaître la même distribution et donc doivent pouvoir être partout interchangeables.

Le recours à la substitution explique, d'une autre façon, la condition d'identité de catégorie grammaticale, déjà évoquée ci-dessus : s'il s'agit de deux catégories grammaticales différentes, l'exercice de commutation est par avance exclu. Mais l'intervention de la commutation a surtout l'avantage d'éviter de s'interroger sur ce qu'on entend par même sens lorsqu'on parle de synonymie. Une définition de ce que signifie avoir le même sens n'est pas une mince affaire et, étant donné les réticences à y faire entrer des considérations référentielles (du type signifier la même chose), difficilement évitables pourtant ${ }^{(18)}$, il peut sembler salutaire de l'aborder par le biais d'une identité de latitudes combinatoires.

Résultat : l'on dispose d'un critère de reconnaissance ${ }^{(19)}$ apparemment assez

(17) La plupart des commentateurs ne parlent pas de variantes libres. Baylon et Fabre (1978: 169) envisagent explicitement le cas.

(18) Voir ici Tamba (1988:84) : «la synonymie a une dimension référentielle évidente, qui ressort de gloses comme : X signifie la même chose que $Y$; X signifie la même chose que $Y »$.

(19) Honeste (2007: 162) mentionne que «le critère d'identification » de la synonymie «vraie » est « la substituabilité totale dans tous les contextes». 
fiable de la synonymie absolue, dont l'évidence est telle qu'il est bien souvent présenté comme un critère définitoire même de la synonymie absolue. Ulmann, cité par Lyons (1970 : 342), formule explicitement le lien entre identité de sens et commutation totale sans altération de ce sens : «On ne peut appeler synonymes que les mots qui peuvent se substituer l'un à l'autre dans tout contexte sans le moindre changement dans la portée cognitive ou affective ». Bayon et Fabre (1978: 169) rappellent que « le critère distributionnel définit la synonymie comme suit : "les vrais synonymes sont mutuellement substituables dans tous leurs environnements" ", affirmation qui se retrouve dans l'équation posée par Danell (1995:9) : « synonymie = identité sémantique ou emplois identiques » ou dans celle de Baylon et Fabre (1978 : 168) : «les synonymes parfaits ou variantes sémantiques complètement libres ». En partant de l'idée que le sens « is anything which affects the contextual normality of lexical items in grammatically well-formed sentential contexts », Cruse (2000:157) définit les synonymes absolus comme des mots qui sont équinormaux dans tous les contextes: $\mathrm{X}$ et $\mathrm{Y}$ sont des synonymes absolus si, dans tous les contextes où X est pleinement approprié, Y l'est aussi, si, dans tous les contextes où $\mathrm{X}$ est légèrement déviant, Y l'est aussi et si, dans tous les contextes où $\mathrm{X}$ est totalement anormal, Y l'est aussi.

La conséquence est attendue : le critère qui reconnaît ou définit la synonymie absolue représente en même temps le facteur qui la condamne, parce qu'une telle interchangeabillité ne se trouve évidemment pas, comme le constatent sans surprise tous les spécialistes. Ainsi, par exemple, Mortureux (1997 : 81) rejette la synonymie entre trouver etjuger et entre retarder et remettre, parce qu'ils ne sont pas interchangeables dans tous leurs emplois : « [...] dans chacun de leurs emplois, la plupart des mots peuvent commuter avec un autre sans que cela affecte beaucoup le sens de l'énoncé : par exemple, j'ai retardé / remis mon départ ; j'ai trouvé / jugé ce problème facile. Les verbes retarder et remettre, d'une part, trouver et juger, d'autre part, ne sont pas pour autant synonymes car cette commutation n'est possible que dans un nombre de cas limité ; ainsi j'ai trouvé ce problème dans un vieux manuel ne la tolère pas, pas plus que j'ai remis ce paquet à son destinataire ou l'affaire est jugée ». Honeste (2007 : 170) se sert également, entre autres, d'un tel argument pour bannir, d'un triple point de vue (cognitif, linguistique et discursif), la notion de synonymie-identité. Elle part de la synonymie qu'on prête habituellement à perdre et à égarer "parce qu'ils renvoient à la même expérience du monde », synonymie que prouverait leur interchangeabilité dans des énoncés tels que :

\section{Jean a perdu sa montre}

Jean a égaré sa montre

«Il est facile, ajoute-t-elle (2007 : 107) alors, de trouver des contextes d'emploi dans lesquels ces deux mots sont beaucoup moins facilement substituables, comme :

Jean vient de perdre sa montre. ? Jean vient d'égarer sa mère

Jean est perdu dans ses pensées. ? Jean est égaré dans ses pensées ».

Même des synonymes comme sofa et settee ou encore pullover et sweater, pour lesquels, selon Cruse (2004 : 157), il semble difficile de trouver des contextes différenciateurs, échouent finalement à l'épreuve de la commutation, ainsi que l'a vérifié Cruse lui-même auprès de ses étudiants : «However, even for these items, in a typical class of students, a sizeable minority will find contexts which for them are discriminating » (Cruse, 2000 : 157). Nous avons déjà donné ci-dessus un autre 
exemple de cette difficulté d'avoir, même pour de sérieux candidats à la synonymie absolue, une interchangeabilité qui soit vraiment totale. Le sens connotatif « domaine sportif » assigné à vélo et non à bicyclette se trouve, comme nous l'avons vu, légitimé par Gaudin et Guespin (2000 : 178) au moyen de différences d'emploi telles que vélo de course opposé à ? bicyclette de course.

Il n'y a donc rien de surprenant si un grand nombre de sémanticiens rejettent la synonymie absolue au nom d'une interchangeabilité qui n'est pas totale et optent pour une synonymie moins stricte, se manifestant ou se définissant par une interchangeabilité restreinte à certains environnements ou emplois seulement. La synonymie « vraie », celle de la substituabilité parfaite, étant « un phénomène strictement inobservable dans la langue » (Honeste, 2007: 162), il n'y a aucune raison de s'y intéresser sérieusement.

\subsection{Ce n'est pas seulement du sens que l'on commute !}

Et pourtant! Il nous semble que c'est aller un peu vite en besogne que d'éliminer la synonymie absolue en avançant comme raison principale que la commutation « absolue » n'est pas possible. L'argument ne nous semble pas recevable, parce qu'il méconnaît, chose que nous avons déjà eu l'occasion de souligner plusieurs fois, que ce n'est pas seulement le sens qui est concerné, mais l'unité lexicale dans sa globalité. Ce n'est pas simplement « un sens » ou « du sens » que l'on commute, mais bien des unités lexicales, c'est-à-dire des unités possédant une forme et un sens.

Toutefois, avant d'aborder le problème à ce niveau-là, il convient de faire une mise au point sur le « lieu » même de la synonymie, parce que cette mise au point permet déjà d'éliminer un type de contre-exemples souvent utilisé dans la littérature. La synonymie se localise au niveau du sens et donc, si un lexème est polysémique, il ne faut envisager la synonymie qu'au niveau de chacun de ses sens. Et, par conséquent aussi, la substitution ne doit être envisagée qu'au niveau de chaque acception et non pour l'ensemble des différents sens relevés. Il est évident que si l'on essaie de substituer un synonyme d'un polysème $\mathrm{X}$ dans son sens 1 à $\mathrm{X}$ pris dans son sens 2 l'échec est garanti, comme le prouve l'utilisation actuelle, grandissante, de la synonymie pour démontrer l'existence de la polysémie (François, 2007 et François, Victorri et Manguin, 2005). Les deux exemples invoqués par Mortureux et Honeste (voir supra) pour illustrer l'impossibilité d'avoir une substituabilité parfaite sont en conséquence non pertinents, puisque dans les contextes où échoue la commutation d'un vocable à l'autre, le vocable qui peut s'y employer présente un sens différent de celui qu'il avait dans les contextes où la commutation réussissait. Trouver, remettre et juger n'ont plus le même sens en passant de j'ai remis mon départ et j'ai trouvé / jugé ce problème facile à j'ai trouvé ce problème dans un vieux manuel, j'ai remis ce paquet à son destinataire et l'affaire est jugée. Il en va de même avec le couple perdre / égarer de Honeste : lorsque perdre prend le sens glosé par le Petit Robert comme «être séparé de quelqu'un par la mort », il n'est à l'évidence plus question d'essayer de le remplacer par égarer et donc l'argument contre la non-interchangeabilité totale des synonymes perdre et égarer manque sa cible. Précisons pour éviter toute erreur d'interprétation, que ce n'est pas pour autant, bien entendu, qu'on a prouvé qu'ils sont synonymes ! Ça, c'est encore une autre histoire ! L'important à ce niveau est de retenir que l'interchangeabilité n'est à comprendre, s'il s'agit de lexèmes polysémiques, qu'au seul niveau de l'acception (ou du sémème) et non au niveau de toute la pluralité de 
sens. Et si donc on examine le critère de l'impossibilité d'interchangeabilité totale, c'est bien évidemment uniquement à ce niveau-là qu'il faut se placer.

Cette limite étant tracée, essayons de voir pourquoi l'inexistence d'une substituabilité parfaite n'est pas un critère pertinent pour se débarrasser de la synonymie-« identité de sens ». Lyons (1970) et Martin (1976) ont souligné tous deux que l'identité de sens et l'interchangeabilité étaient deux phénomènes qu'il fallait distinguer ${ }^{(20)}$. Pour Lyons (1970:343), c'est en effet une « illusion » que de « formuler une définition de la synonymie absolue qui repose sur l'idée que l'équivalence complète et l'interchangeabilité totale sont nécessairement liées ». En conséquence, Lyons et Martin proposent une typologie des synonymes où l'équivalence de sens, complète ou incomplète, se trouve séparée de la substituabilité, totale ou partielle. Nous n'examinerons pas ici la pertinence des différents cas possibles de synonymie ainsi obtenus. L'important pour nous est la dissociation opérée entre identité de sens et commutation totale et la possibilité qui en découle d'avoir une identité de sens complète sans interchangeabilité totale correspondante. Autrement dit, la possibilité de continuer à dire que bicyclette et vélo sont des synonymes absolus, alors même qu'ils ne commutent pas dans tous les contextes possibles.

Faisons un pas de plus et expliquons pourquoi le lien obligatoire entre l'identité de sens et la commutation dans tout contexte n'est pas légitime. La raison, déjà annoncée ci-dessus, est que ce n'est pas seulement du sens que l'on commute, mais des mots pris dans leur unité sémiotique formelle et sémantique (une acception) et que donc la bonne formation d'une unité insérée dans un contexte ne dépend pas uniquement de son sens, mais également de ses propriétés formelles et des propriétés qui lui sont propres en tant qu'unité sémiotique et dont nous avons pu mesurer ci-dessus l'importance lors de la question de la distinction sens dénotatif / sens connotatif. Ce qui se trouve présent dans un contexte, c'est bien une unité lexicale et ce fait, que l'on ne peut nier, a pour conséquence d'exclure par avance l'interchangeabilité totale entre deux unités lexicales différentes. Il suffit en effet que le contexte ait un rapport avec, c'est-à-dire active, ce qui fait que deux mots de sens identiques sont précisément des mots différents pour que la substitution de l'un à l'autre ne soit a priori plus possible. Nous voulons dire par là que, théoriquement, l'interchangeabilité totale n'est pas possible. Du coup, on ne peut plus, bien évidemment, l'utiliser comme argument pour expulser du lexique les synonymes absolus, en prétextant qu'ils n'ont pas de sens totalement identique puisqu'ils ne commutent pas partout.

Pour montrer que la substituabilité totale n'est a priori pas possible, nous reprendrons d'abord l'exemple du couple pharamineux / faramineux qui ne représente, comme souligné ci-dessus, qu'une simple variante formelle et qui, parce qu'il ne constitue qu'un seul mot, ne peut prétendre représenter un cas de synonymie. Or, cette petite différence de forme scripturale interdit également par avance l'interchangeabilité totale des deux formes à l'écrit, puisque, dans les énoncés qui mettent en avant les deux formes et leur différence, la substitution de l'un à l'autre est évidemment exclue :
Ça s'écrit pharamineux ou faramineux
? Ça s'écrit faramineux ou faramineux
? Ça s'écrit pharamineux ou pharamineux

(20) Baylon et Fabre (1978: 169) précisent aussi que le critère distributionnel de la substituabilité dans tous les contextes «ne semble pas nécessairement en rapport étroit avec la similitude de sens". 
On notera en passant que l'échec de la substitution ne va pas entraîner un verdict de différenciation qui porterait sur autre chose que la variation formelle $p h / f$ de départ.

Si on passe aux situations de synonymie, c'est-à-dire celles qui impliquent véritablement deux mots différents, la substitution se retrouve par avance bloquée dans tous les énoncés qui présentent les deux mots différents en établissant ou mettant en jeu leur identité. On comprend facilement pourquoi : si on remplace l'un des deux termes par l'autre, l'énoncé obtenu perd toute sa pertinence, puisqu'il revient à établir ou à mettre en jeu l'identité avec lui-même du terme mis à la place de l'autre. On retrouve là le « puzzle» référentiel d'identité familier aux philosophes du langage obligés d'expliquer pourquoi $a$ est $b$ n'est malgré tout pas identique à $a$ est $a$ et $b$ est $b^{(21)}$ :
Les vélos, c'est des bicyclettes
? Les vélos, c'est des vélos
? Les bicyclettes, c'est des bicyclettes
Les brugnons ou nectarines...
? Les nectarines ou nectarines...
? Les brugnons ou brugnons...
Les brugnons, c'est-à-dire les nectarines,...
? Les brugnons, c'est-à-dire les brugnons,...
? Les nectarines, c'est-à-dire les nectarines,...

et l'énigme des énoncés indirects donnant lieu à l'opposition transparence / opacité $^{(22)}$ :

La cliente voulait savoir si les brugnons, c'étaient des nectarines

? La cliente voulait savoir si les brugnons, c'étaient des brugnons

? La cliente voulait savoir si les nectarines, c'étaient des nectarines

On nous accusera sans doute de chercher midi à quatorze heures - en l'occurrence ce serait plutôt « chercher midi à midi »-nous en convenons fort volontiers, mais il nous semble que ce premier type de contexte rétif à toute commutation a l'avantage de montrer que le simple fait d'avoir, en cas de synonymie, deux unités lexicales différentes donne déjà lieu à des contraintes d'emploi différentes.

Le deuxième type de contextes fermés a priori à la substitution est constitué par les énoncés qui activent, d'une manière ou d'une autre, des propriétés non sémantiques associées à l'unité lexicale utilisée. La commutation échoue alors tout logiquement, si le mot mis à la place du premier ne présente pas ces propriétés. Les propriétés en question sont en gros celles de la forme et celles que nous avons fait sortir ci-dessus du sens connotatif pour les reporter sur le mot lui-même. Elles peuvent être activées parce que le mot est réellement présent dans le discours. Comme il se montre ou s'exhibe (Récanati, 1979, Kleiber, 1984 et Authier-Revuz, $1995^{(23)}$, on peut toujours renvoyer réflexivement à sa forme, à ses propriétés de

(21) Ce qui montre que la solution de Frege, qui consiste à dire que la référence est identique, mais le sens différent, n'est pas tout à fait correcte, puisque si elle correspond aux cas où une des deux expressions au moins est une description définie, elle est inappropriée pour expliquer des énoncés mettant aux prises deux unités lexicales.

(22) Le problème est en fait beaucoup plus complexe (voir Kleiber, 1981 : Ch. IX).

(23) Rey-Debove (1978) parle de connotation autonymique ou connotation langagière réflexive pour marquer que le signe a comme connotation son autonyme. 
signe par des procédés divers, le plus souvent métalinguistiques ${ }^{(24)}$. Nous ne donnerons que quelques exemples ci-dessous, mais qui suffisent à montrer, d'une part, que le renvoi réflexif effectué empêche la substitution du mot employé par un mot synonyme et, d'autre part, que les principaux facteurs rassemblés sous l'étiquette de signifié connotatif peuvent se retrouver ici, ce qui prouve d'une autre manière qu'il ne s'agit pas de propriétés du sens, mais de propriétés attachées au mot :

Les pompiers sont ainsi appelés parce qu'ils utilisent des pompes à eau pour éteindre l'incendie

? Les soldats du feu sont ainsi appelés parce qu'ils utilisent des pompes à eau pour éteindre l'incendie

Une bicyclette, comme son nom l'indique, a deux roues ${ }^{(25)}$

? Un vélo, comme son nom l'indique, a deux roues

J'en veux septante, comme on dit en Belgique

? J'en veux soixante-dix, comme on dit en Belgique

C'est super, comme disent les jeunes aujourd'hui

? C'est formidable, comme disent les jeunes aujourd'hui

Sa meuf, pour parler comme en banlieue,...

? Sa femme, pour parler comme en banlieue,...

Il a une rhinite, pour utiliser un mot de toubib,...

? Il a un rhume, pour utiliser un mot de toubib,...

Il s'est cassé la pipe, pour parler de façon expressive / imagée

? Il est mort, pour parler de façon expressive / imagée

On pourrait encore citer les jeux de mots reposant sur la forme du mot, qui interdisent, bien entendu, toute substitution synonymique ( $c f$. par exemple le titre rock and grolles du Canard Enchaînédu mercredi 17 décembre 2008 qui perd toute pertinence si on remplace grolles par chaussures ou souliers), mais l'essentiel nous semble démontré : l'interchangeabilité totale n'est pas possible et donc ne peut pas constituer un argument pour rejeter la synonymie-« identité de sens ».

\section{Conclusion}

Le moment est venu de conclure : les trois principaux arguments avancés habituellement pour rejeter la synonymie absolue ne s'avèrent finalement pas pertinents. Le premier, parce que la synonymie-«identité de sens » n'est pas un luxe inutile, embarrassant, qui contrevient au principe d'opposition et d'économie linguistique, mais présente au contraire une fonctionnalité discursive précieuse et efficace. Le second, parce que le signifié connotatif souvent invoqué pour rejeter toute idée de synonymes à sens identique ne correspond pas à du « sémantique », mais représente des propriétés attachées au mot en tant qu'unité sémiotique. Le

(24) Dont notamment les guillemets par exemple.

(25) Si Bicyclette a trois roues est beaucoup plus difficile à dire que vélo à trois roues, comme Sylvain Kahane l'a fait observer à Polguère (2008 : 150), c'est précisément à cause de la composition encore transparente de bicyclette. 
troisième, nous venons de le voir, parce que l'interchangeabilité « absolue » est a priori impossible, de telle sorte qu' on ne peut s'en servir pour bannir la synonymie « absolue ».

Bien évidemment, il faut définir cette synonymie absolue autrement que par la simple mention de l'identité sémantique, même si cette notion nous semble intuitivement à l'œuvre dans maints raisonnements linguistiques, qui n'ont pas forcément à voir avec la synonymie. S'ouvre ici la problématique de l'implication réciproque, défendue par Lyons (1970) et Martin (1976), qui, certes, ne va pas sans difficulté, mais qui trace une piste explicative qu'on aurait tort de ne pas suivre sérieusement et qu'il convient de relier à la question de la substituabilité. S'ouvre aussi et surtout la problématique des parasynonymes ou synonymes approximatifs, c'est-à-dire des termes dont le sens n'est pas totalement identique, mais que l'on a néanmoins intérêt à rapprocher plutôt qu'à séparer. C'est là que nous reprendrons pour terminer la formule de Cruse mise en exergue à notre article : c'est vrai, il y a encore du pain sur la planche en matière de synonymie !

\section{Bibliographie}

AUTHIER-REVUZ, J. (1995) : Ces mots qui ne vont pas de soi. Boucles réflexives et non-coïncidence du dire, Paris, Larousse.

BALDINGER, K. (1984) : Vers une sémantique moderne, Paris, Klincksieck.

BATTEUX, M., 2000, Die französische Synonymie im Spannungsfeld zwischen Paradigmatik und Syntagmatik, Frankfurt am Main, Peter Lang.

BAYlon, C. et FABre, P. (1978) : La sémantique, Paris, Nathan.

BERLAN, F. (2007) : «Peut-on concilier la notion de valeur et le recours à l'étymologie en synonymie distinctive ? », Le Français Moderne, 75 : 1, 41-66.

BRÉAL, M. (1897) : Essai de sémantique, Paris, Hachette.

BREKLE, H.E. (1974) : Sémantique, Paris, Armand Colin.

CRUSE, D. A. (2000) : Meaning in Language, Oxford, Oxford University Press.

DANELL, K.-J. (1995) : Le phénomène de concurrence en français moderne, Uppsala, Swedish Science Press.

Dumarsais, Des Tropes ou des différents sens, 1730, ch.3, XII, 231-237, éd. par Douay-Soublin, F., Paris, Flammarion 1988.

FRANÇOIS, J. (2007) : Pour une cartographie de la polysémie verbale, Leuven-Paris, Peteters.

FrançOis, J., Victorri, B. et MAnguin, J.-L. (2005) : «Polysémie adjectivale et synonymie », in Soutet, O. (éd.), La polysémie, Paris, Presses de l’Université de Paris-Sorbonne, 175-188.

FREI, H. (1929) : La grammaire des fautes, Bellegarde.

GAUDIN, F. et GUESPIN, L. (2000) : Initiation à la lexicologie française, Bruxelles, Duculot.

GAUGER, H.M. (1972) : Zum Problem der Synonymie, Tübingen, Tübinger Beiträge zur Linguistik. 
GIRARD, G. (Abbé) (1736) : Les synonymes françois, leurs différentes significations et le choix qu'il faut en faire pour en parler avec justesse, Paris, Veuve d'Houry.

HONESTE, M.-L. (2007) : «Entre ressemblance et différence : synonymie et cognition », Le Français Moderne, 75 : 1, 160-173.

KLEIBER, G. (1981) : Problèmes de référence. Descriptions définies et noms propres, Paris, Klincksieck.

— (1984) : «"MONSIEUR AUGUSTE” est venu : théorie naïve, théorie de la métanomination et théorie "réductionniste" ", in Kleiber, G. (éd.), Recherches en pragma-sémantique, Paris, Klincksieck, 215-231.

LEEMAN, D. (2002) : «Me est un autre», in Anis, J., Eskénazi, A. et Jeandillou, J.-F. (éds), Le signe et la lettre. Hommages à Michel Arrivé, Paris, L'Harmattan, 349-358.

Lehmann, A. \& MARTin-Berthet, F. (1998) : Introduction à la lexicologie. Sémantique et morphologie, Paris, Dunod.

LYONS, J. (1970) : Linguistique générale, Paris, Larousse.

MARTIN, R. (1976) : Inférence, antonymie et paraphrase, Paris, Klincksieck.

MEL'CUK, I., Clas, A. et Polguère, A. (1995) : Introduction à la lexicologie explicative et combinatoire, Louvain-la-Neuve, Duculot.

MitTeRAND, H. (1963) : Les mots français, Paris, PUF.

MORTUREUX, M.-F. (1997) : La lexicologie entre langue et discours, Paris, SEDES.

Neveu, F. (2004) : Dictionnaire des sciences du langage, Paris, Armand Colin.

NYCKEES, V. (1998) : La sémantique, Paris, Belin.

Picoche, J. (1993) : Didactique du vocabulaire français, Paris, Nathan.

POlguÈre, A. (2008) : Lexicologie et sémantique lexicale; Montréal, Les Presses de l'Université de Montréal.

RÉCANATI, F. (1979) : La transparence et l'énonciation, Paris, Seuil.

REY-DEBOVE, J. (1978) : Le métalangage. Etude linguistique du discours sur le langage, Paris, Le Robert.

- (1997) : «La synonymie ou échange de signes comme fondement de la sémantique », Langages, 128.

SANCHEZ, J. et CADIOT, P. (2007) : « Le cas des substantifs construits », Le Français Moderne, $75: 1,114-140$.

SIOUFFI, G. et VAN RAEMDONCK, D. (1999) : 100 fiches pour comprendre la linguistique, Rosny, Bréal.

TAMBA, I. (1988) : La sémantique, Paris, PUF, $5^{\mathrm{e}}$ édition refondue 2005.

Touratier, C. (2000) : La sémantique, Paris, Armand Colin.

Ullmann, S. (1952) : Précis de sémantique française, Berne, Francke, 4 édition 1969.

- (1962) : Semantics : an Introduction to the Science of Meaning, Oxford ; Blackwell.

- (1964) : Language and Style, Oxford, Blackwell.

VON Wartburg,W. (1969) : Problèmes et méthodes de la linguistique, Paris, PUF ( $3^{\mathrm{e}}$ édition). 